NOUVELle

\section{Tau et troubles cognitifs}

\section{Un rôle pour les lymphocytes T}

Cyril Laurent ${ }^{1}$, Guillaume Dorothée ${ }^{2,3}$, Stéphane Hunot ${ }^{4-7}$, Elodie Martin ${ }^{4-7}$, Cécile Delarasse ${ }^{4-7}$, Luc Buée ${ }^{1}$, David Blum ${ }^{1}$

\section{La maladie d'Alzheimer}

La maladie d'Alzheimer (MA) est la démence la plus fréquente qui atteint graduellement les fonctions cognitives. La MA se définit par la présence conjointe de deux lésions neuropathologiques: les plaques séniles, qui correspondent à des dépôts extracellulaires de formes fibrillaires du peptide amyloïde $A \beta$, et les dégénérescences neuro-fibrillaires, qui sont des agrégats intra-neuronaux de protéines Tau hyper-phosphorylées (nommée pathologie Tau). Ces derniers sont également retrouvés dans un large spectre de pathologies neurologiques regroupées sous le nom de Tauopathies [1]. Ces lésions cérébrales sont associées à des réponses immunitaires neuroinflammatoires chroniques innées, sous la forme d'activations microgliale et astrocytaire, qui sont suspectées de participer au développement physiopathologique de la MA [2]. En revanche, le rôle de l'immunité adaptative demeure énigmatique et a été essentiellement étudié sur le versant amyloïde de la MA.

\section{La protéine Tau}

La protéine Tau, exprimée principalement par les neurones, appartient à la famille des protéines associées aux microtubules. Sa liaison à la tubuline via ses domaines de liaison carboxy-terminaux, stabilise le réseau microtubulaire et favorise le transport axonal [1]. Tau peut subir plusieurs modifications post-traductionnelles dont la phosphorylation, qui est majoritairement la plus fréquente puisque plus de 80 sites potentiels ont été identifiés dans la protéine. Dans les conditions pathologiques, un déséquilibre entre l'activité des kinases et des phosphatases modifiant Tau est à l'origine d'une élévation anormale des formes hyper-phosphorylées de la protéine, favorisant son oligomérisation et son agrégation. La propagation stéréotypée et hiérarchique de la pathologie Tau selon les stades de Braak, est corrélée aux altérations cognitives chez les patients atteints de MA [3], suggérant un rôle instrumental dans les dysfonctions synaptiques sous-jacentes. Cette propagation se développe selon un mode trans-synaptique de type prion, dont les mécanismes ne sont pas clairement élucidés, mais qui font intervenir une libération de la protéine Tau par des exosomes, des ectosomes ${ }^{1}$ et d'autres structures comme $(\rightarrow$ ) Voir la Nouvelle celles de type nano- de F. Clavaguera et al., $m / s \mathbf{n}^{\circ} 2$, tube [4-6] $(\rightarrow)$ février 2010, page 121 Des travaux récents démontrent également le rôle important des cellules microgliales dans ce phénomène [5-7].

Immunité innée, maladie d'Alzheimer et pathologie Tau

Les études pan-génomiques, réalisées avec l'objectif d'identifier des facteurs de risques associés au développement de la MA, ont mis en évidence la présence de différents variants de gènes liés aux fonctions immunes et microgliales.

\footnotetext{
${ }^{1}$ Les ectosomes (ou microparticules) sont (comme les exosomes) des vésicules extracellulaires produites par une cellule ou un organisme à partir d'une membrane.
}

On retrouve parmi ceux-ci, notamment, l'antigène CD33, le récepteur du complément de type l (CRI), la clusterine ${ }^{2}$ (clu), la protéine membranaire ms4a (membrane-spanning 4-domains subfamily 4) ou encore le récepteur trem2 (triggering receptor expressed on myeloid cells 2) [7, 8]. La participation du système immunitaire dans la progression de la MA a également été suggérée par des études épidémiologiques faisant le lien entre risque de survenue de la MA et utilisation prolongée d'anti-inflammatoires non-stéroïdiens (AINS). Ces études demeurent cependant encore contradictoires. La microglie semble jouer un rôle majeur dans la physiopathologie de la MA, notamment en lien avec l'accumulation de peptides $A \beta$. Ces derniers sont détectés par les cellules microgliales via des récepteurs de danger présents à la surface des cellules, tels que les Toll-like receptors (TLR) de type 2 et 4, ou intracellulaires comme les Nod-like receptors (NLR), constituants de l'inflammasome et impliqués dans la libération d'interleukine-1 beta (IL-1 $\beta$ ) [2]. Les réponses microgliales paraissent jouer un rôle complexe au cours de la maladie. Elles auraient un rôle bénéfique aux stades précoces via l'élimination des agrégats amyloïdes par phagocytose, puis une fonction délétère en contribuant au processus pathologique

${ }^{2}$ La clusterine est une glycoprotéine protectrice de l'intégrité cellulaire. 


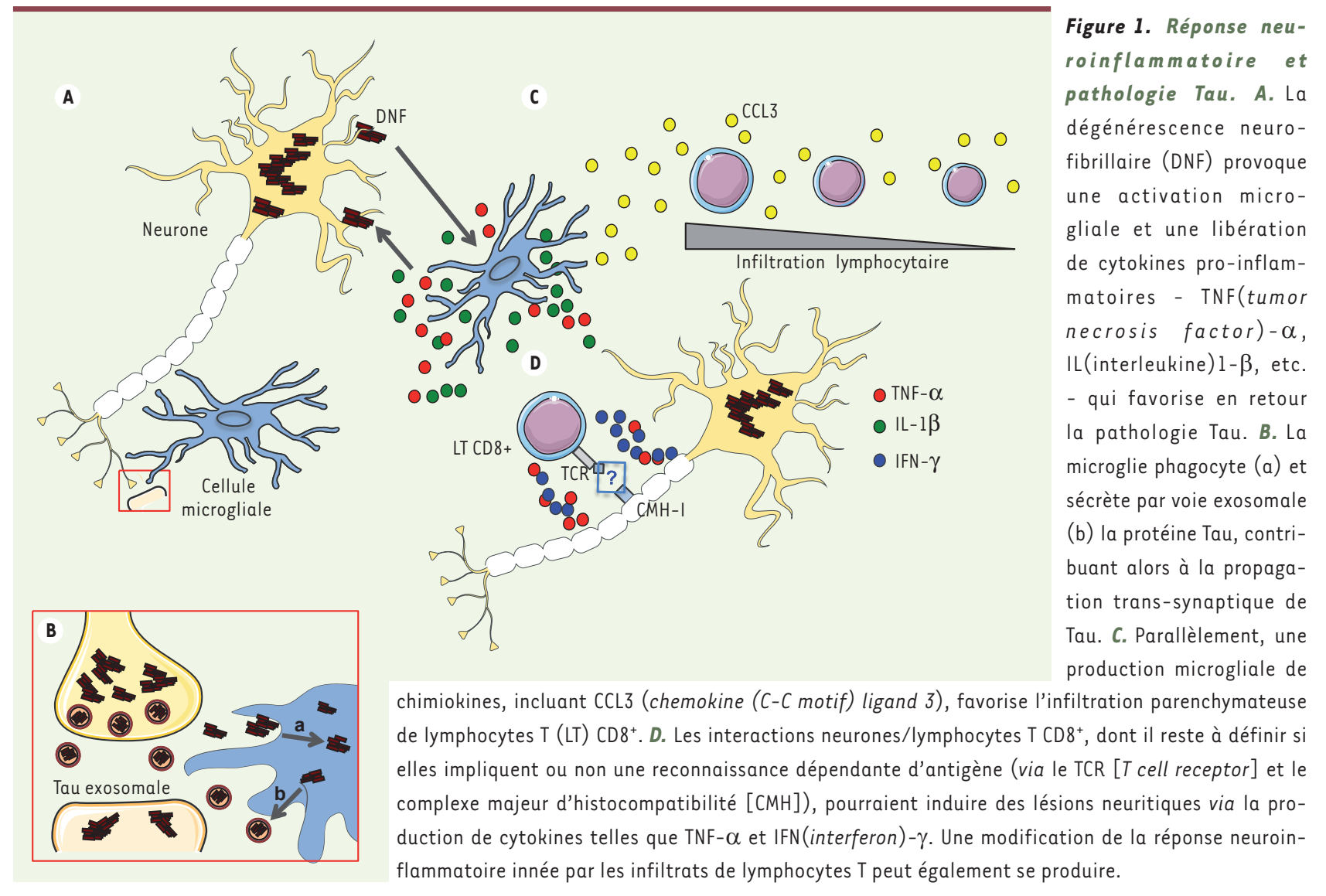

[2]. Ce rôle bi-phasique de la microglie a été récemment suggéré par imagerie cérébrale lors de la progression de la maladie chez les patients atteints de MA $[8,9]$. La relation entre immunité innée et pathologie Tau a été peu étudiée. Toutefois, les travaux réalisés jusqu'à présent s'accordent pour suggérer que les dégénérescences neurofibrillaires intraneuronales provoquent également une activation microgliale $[9,10]$ qui, en retour, favorise l'hyper-phosphorylation, l'agrégation et la sécrétion de la protéine Tau [7, 11]. Ces changements créent donc un cercle vicieux qui contribuerait au développement des troubles cognitifs. Les mécanismes sous-jacents demeurent peu connus, mais il est logique de penser que les formes extracellulaires de Tau joueraient un rôle important.

Pathologie Tau : de l'immunité innée à l'immunité adaptative

En s'appuyant sur un modèle murin développant des lésions neurofibrillaires (les souris transgéniques Thy-Tau22 ${ }^{3}$ ), nous avons confirmé l'association entre pathologie Tau et immunité innée. Nous avons également démontré l'existence d'une réponse chimiokinique d'origine microgliale, en corrélation avec le développement progressif de la pathologie Tau hippocampique et des troubles mnésiques associés, dans ce modèle de Tauopathie. La réponse chimiokinique se caractérise en particulier par une production précoce de la chimokine CCL3/ MIPl $\alpha$ (C-C-motif-ligand 3/ macrophage inflammatory protein 1-alpha) [12]. Les chimiokines sont des molécules de type chimio-attractant qui facilitent notamment la migration et l'infiltration cérébrale des leucocytes circulants via les plexus choroïdes ou à travers la barrière hémato-encéphalique $(B H \varepsilon)$, notamment en situation patho-

\footnotetext{
${ }^{3}$ Ces souris expriment une protéine Tau humaine (IN4R) mutée sur deux résidus (G272V et P301S), favorisant son agrégation, sous le contrôle du promoteur neuronal Thyl.2.
}

logique. Nos données montrent que la production de CCL3 par les cellules microgliales est associée à une infiltration des lymphocytes $T$, essentiellement CD8 ${ }^{+}$, dans le parenchyme cérébral des souris Thy-Tau22, sans perte d'intégrité de la BHE. À l'inverse, aucune infiltration de monocytes d'origine périphérique n'est observée. Une infiltration parenchymateuse de lymphocytes $\mathrm{T} \mathrm{CD8}^{+}$est également retrouvée dans le cerveau de patients atteints d'une Tauopathie génétique (ou dégénérescence fronto-temporale, frontotemporal dementia with parkinsonism-17, [FTDP-17]), renforçant l'idée d'un lien entre la pathologie Tau et l'infiltration lymphocytaire. Nos observations sont par ailleurs cohérentes avec des travaux antérieurs démontrant une corrélation entre l'infiltration parenchymateuse de lymphocytes $\mathrm{TCD}^{+}$et la pathologie Tau dans le cerveau de patients atteints de MA [12, 13]. Mais qu'en est-il du rôle de ces infiltrats? 
Impact de l'infiltration lymphocytaire sur le développement physiopathologique

Afin d'évaluer l'impact fonctionnel de l'infiltration lymphocytaire dans le modèle murin Thy-Tau22, nous avons provoqué une déplétion massive des lymphocytes T qui n'affecte pas les lymphocytes B. Nos données montrent que l'administration chronique (deux fois par mois) d'un anticorps anti-CD3 - initiée à un moment où l'infiltration de lymphocytes $T$ n'est pas présente et se poursuivant jusqu'à un stade avancé de la pathologie - réduit drastiquement l'infiltration parenchymateuse des lymphocytes $\mathrm{T} C D 8^{+}$. De manière extrêmement intéressante, cette déplétion des cellules T améliore les performances mnésiques des animaux tout en réduisant la réponse neuroinflammatoire hippocampique. Toutefois, ce traitement n'affecte pas la phosphorylation et l'accumulation de protéines Tau pathologiques. Ces observations suggèrent donc que les infiltrats lymphocytaires associés au développement de la pathologie Tau ont un effet délétère sur les mécanismes de plasticité synaptique qui sous-tendent la mémoire [12]. Nous avons récemment mis en évidence, dans un modèle de pathologie amyloïde, les effets bénéfiques d'une population particulière de lymphocytes $T$ CD4 ${ }^{+}$, les lymphocytes $T$ régulateurs, qui améliorent les performances mnésiques indépendamment d'un effet sur la pathologie amyloïde, mais en association avec une modulation de la réponse microgliale [14]. L'impact des infiltrats de lymphocytes $T$ sur la plasticité synaptique pourrait donc être de nature indirecte, via une modification de la réponse neuroinflammatoire innée. Par ailleurs, les lymphocytes T CD8 ${ }^{+}$ parenchymateux pourraient aussi directement affecter les fonctions neuronales et la plasticité synaptique (Figure I). $\varepsilon n$ effet, une interaction entre les neurones et les lymphocytes $T \operatorname{CD}^{+}$a été décrite, ces derniers pouvant induire des lésions neuritiques, voire la mort neuronale, et conduire à des altérations de la plasticité via notamment la production de cytokines (TNF[tumor necrosis factor]- $\alpha$, IFN [interferon] $\gamma$ par exemple [15]). Les mécanismes mis en jeu dans le contexte de la MA, et notamment en lien avec la pathologie Tau, demeurent encore à explorer.

\section{Conclusion}

Nos travaux contribuent à souligner le rôle de l'immunité cellulaire adaptative dans la physiopathologie de la MA. Ils sont les premiers à impliquer une infiltration lymphocytaire $T$ dans les troubles synaptiques et mnésiques liés à la protéine Tau. L'identité précise des souspopulations de lymphocytes $T$ participant au processus ainsi que les mécanismes cellulaires et moléculaires mis en jeu restent à préciser afin de comprendre le rôle physiopathologique de ces cellules et ainsi développer des approches immunothérapeutiques innovantes pour le traitement de la MA et des Tauopathies. $\diamond$

Tau and cognitive disorders: a role for Tlymphocytes

\section{LIENS D'INTÉRÊT}

Les auteurs déclarent n'avoir aucun lien d'intérêt concernant les données publiées dans cet article.

\section{RÉFÉRENCES}

1. Sergeant N, Bretteville A, Hamdane M, et al. Biochemistry of tau in alzheimer's disease and related neurological disorders. Expert Rev Proteomics 2008; $5: 207-24$.

2. Heneka MT, Carson MJ, દl Khoury J, et al. Neuroinflammation in Alzheimer's disease. Lancet Neurol $2015 ; 14: 388-405$.

3. Duyckaerts C, Bennecib M, Grignon Y, et al. Modeling the relation between neurofibrillary tangles and intellectual status. Neurobiol Aging 1997 ; 18 : 267 73.

4. Tardivel M, Begard S, Bousset $L$, et al. Tunneling nanotube (tnt)-mediated neuron-to neuron transfer of pathological tau protein assemblies. Acta Neuropathol Commun 2016 ; 4 : 117.

5. Clavaguera F, Goedert M, Tolnay M. Induction et propagation de la pathologie par la protéine tau chez un modèle murin de la maladie d'Alzheimer. Med Sci (Paris) $2010 ; 26: 121-4$.

6. Dujardin S, Bégard S, Caillierez R, et al. Ectosomes: a new mechanism for non-exosomal secretion of Tau protein. PLoS One 2014 ; 9 : el00760.

7. Asai H, Ikezu S, Tsunoda S, et al. Depletion of microglia and inhibition of exosome synthesis halt tau propagation. Nat Neurosci 2015 ; 18 : 1584-93.

8. Tosto G, Reitz C. Genome-wide association studies in alzheimer's disease: a review. Curr Neurol Neurosci Rep $2013 ; 13: 381$.
9. Fan Z, Brooks DJ, Okello A, et al. An early and late peak in microglial activation in alzheimer's disease trajectory. Brain 2017 ; 140 : 792-803.

10. Yoshiyama Y, Higuchi M, Zhang B, et al. Synapse loss and microglial activation precede tangles in a p301s tauopathy mouse model. Neuron 2007 ; 53 : 337-51.

11. Bhaskar K, Konerth M, Kokiko-Cochran ON, et al. Regulation of tau pathology by the microglial fractalkine receptor. Neuron $2010 ; 68$ : 19-31.

12. Laurent C, Dorothee G, Hunot $S$, et al. Hippocampal $t$ cell infiltration promotes neuroinflammation and cognitive decline in a mouse model of tauopathy. Brain 2017 ; $140: 184-200$.

13. Zotova $\varepsilon$, Bharambe V, Cheaveau M, et al. Inflammatory components in human alzheimer's disease and after active amyloid-beta42 immunization. Brain 2013; 136 : 2677-96.

14. Dansokho C, Ait Ahmed D, Aid S, et al. Regulatory $\mathrm{t}$ cells delay disease progression in alzheimer-like pathology. Brain 2016; 139 : 1237-51.

15. Liblau RS, Gonzalez-Dunia D, Wiendl H, et al. Neurons as targets for $\mathrm{t}$ cells in the nervous system. Trends Neurosci 2013 ; 36 : 315-24.

\section{Modèle de Braak}

Selon le modèle de Braak, l'extension des corps de Lewis ( $\mathrm{CL}$ ) est corrélée au degré de sévérité clinique de la maladie, et suit un chemin ascendant (bulbe olfactif/système entérique-tronc cérébralcortex). Les $\mathrm{CL}$ se développent initialement dans le bulbe olfactif, le noyau dorsal moteur du nerf vague, le système nerveux entérique, les noyaux inférieurs du raphé, le locus cœruleus, et les centres bulbaires et médullaires somato- et viscéro-régulateurs. Cela correspond aux stades 1 et 2, asymptomatique puis prodromal avec hyposmie, signes dysautonomiques, troubles du sommeil et de l'humeur. La phase symptomatique débute au stade 3 , avec atteinte de la substance noire compacte et du noyau pédonculopontin (PPN), et apparition des premiers symptômes moteurs de la triade parkinsonienne. Le cortex est atteint à partir du stade 4 , avec une progression centripète, de sa périphérie au sillon central : cortex temporal trans-entorhinal, entorhinal et hippocampe (stade 4), aires associatives sensitives et préfrontales de haut niveau (stade 5), aires associatives sensitivomotrices de premiers ordre (cortex prémoteur) et cortex sensitivomoteur primaire (stade 6). Les troubles cognitifs et les chutes apparaissent à partir du stade 5 , et la démence au stade 6. 